Appl. Set-Valued Anal. Optim. 3 (2021), No. 1, pp. 109-115

Available online at http://asvao.biemdas.com

https://doi.org/10.23952/asvao.3.2021.1.09

\title{
CONVERGENCE OF ITERATES OF NONLINEAR CONTRACTIVE MAPPINGS
}

\author{
SIMEON REICH ${ }^{1, *}$, ALEXANDER J. ZASLAVSKI $^{2}$ \\ Department of Mathematics, The Technion - Israel Institute of Technology, 32000 Haifa, Israel
}

\begin{abstract}
Recently, we established the convergence of iterates of nonlinear contractive self-mappings of a not necessarily bounded closed subset of a Banach space. In this paper, we extend this result to a larger class of mappings. We also construct an example, which demonstrates that this new class is indeed larger than the analogous classes of mappings, which were studied in the literature.
\end{abstract}

Keywords. Banach space; Contractive mapping; Fixed point.

\section{INTRODUCTION}

For at least fifty-five years, there has been a lot of research activity concerning the fixed point theory of nonexpansive (that is, 1-Lipschitz) mappings. See, for example, $[1,2,3,4,5,6$, 7 , $8,9]$ and the references cited therein. This activity stems from Banach's classical theorem [10] regarding the existence of a unique fixed point for a strict contraction. It also concerns the convergence of (inexact) iterates of a nonexpansive mapping to one of its fixed points. Since that seminal result, many developments have taken place in this field including, in particular, the studies of feasibility, common fixed point problems, monotone and accretive operators, and variational inequalities, all of which find important applications in engineering, medical and the natural sciences $[11,12,13,14,15,16]$.

In our recent work [17], we established the existence of a unique fixed point for nonlinear contractive self-mappings of a bounded and closed subset of a Banach space. More precisely, in $[17,18]$, we considered the following class of nonlinear mappings.

Let $(X,\|\cdot\|)$ be a Banach space, and let $K$ be a bounded, closed and convex subset of $X$. Let $f: X \rightarrow[0, \infty)$ be a continuous function such that $f(0)=0$, the set $f(K-K)$ is bounded, and the following three properties hold:

(i) for each $\varepsilon>0$, there exists $\delta>0$ such that if points $x, y \in K$ satisfy $f(x-y) \leq \delta$, then $\|x-y\| \leq \varepsilon$

(ii) for each $\lambda \in(0,1)$, there is a number $\phi(\lambda) \in(0,1)$ such that

$$
f(\lambda(x-y)) \leq \phi(\lambda) f(x-y) \text { for all } x, y \in K
$$

(iii) the function $(x, y) \mapsto f(x-y), x, y \in K$, is uniformly continuous on $K \times K$.

${ }^{*}$ Corresponding author.

E-mail addresses: sreich@technion.ac.il (S. Reich), ajzasl@technion.ac.il (A.J. Zaslavski).

Received April 28, 2020; Accepted July 30, 2020.

(C)2021 Applied Set-Valued Analysis and Optimization 
Denote by $\mathscr{A}$ the set of all continuous mappings $A: K \rightarrow K$ such that

$$
f(A x-A y) \leq f(x-y) \text { for all } x, y \in K .
$$

For each $A, B \in \mathscr{A}$, set

$$
d(A, B):=\sup \{\|A x-B x\|: x \in K\} .
$$

It is clear that $(\mathscr{A}, d)$ is a complete metric space.

In [18], we established the existence of a set $\mathscr{F}$, which is a countable intersection of open and everywhere dense subsets of $\mathscr{A}$ such that each mapping $C \in \mathscr{F}$ has a unique fixed point and all its iterates converge uniformly to this fixed point.

Note that the classical result of De Blasi and Myjak [19] is a particular case of this result where $f=\|\cdot\|$. As a matter of fact, the mappings defined above can be considered generalized nonexpansive mappings with respect to $f$. Such an approach, where in some problems of Functional Analysis the norm is replaced by a general function, which was used in $[20,21]$ in the study of generalized best approximation problems.

Given a closed subset $S$ of a Banach space $X$ and a point $x \in X$, we considered, in [20, 21], the minimization problem

$$
\min \{f(x-y): y \in S\} .
$$

This problem was studied by many mathematicians mostly in the case that $f(x)=\|x\|$. In this special case, it is well known that if $S$ is convex and $X$ is reflexive, then problem (P) always has at least one solution. This solution is unique when $X$ is strictly convex. In [20,21], we established the generic solvability and well-posedness of problem (P) for a general function $f$.

In [17], we improved the results of [18]. Namely, we introduced the notion of a contractive mapping, and showed that most mappings in $\mathscr{A}$ (in the sense of Baire category) are contractive, and every contractive mapping possesses a unique fixed point and that all its iterates converge to this point uniformly. Note that all these results were obtained for a bounded set $K$.

\section{A FIXED POINT RESULT}

In [22], we extend one of the main results of [17] to unbounded sets. More precisely, we show there that even if $K$ is unbounded, every contractive self-mapping of $K$ possesses a unique fixed point and that all its iterates converge to this point, uniformly on bounded subsets of $K$. Moreover, for this result, we do not need property (ii).

Let $(X,\|\cdot\|)$ be a Banach space and let $K$ be a nonempty and closed subset of $X$. Let $f: X \rightarrow$ $[0, \infty)$ be a continuous function such that $f(0)=0$ and the following two properties hold:

(P1) for each $\varepsilon>0$, there exists $\delta>0$ such that if points $x, y \in K$ satisfy $f(x-y) \leq \delta$, then $\|x-y\| \leq \varepsilon$

(P2) the function $(x, y) \mapsto f(x-y), x, y \in K$, is uniformly continuous on $K \times K$ and for each point $\xi \in K$, the function $f(x-\xi), x \in D$, is bounded for every bounded set $D \subset K$.

Assume that $A: K \rightarrow K$ is a continuous mapping, $\psi:[0, \infty) \rightarrow[0,1]$ is a decreasing function satisfying

$$
\psi(t)<1 \text { for all } t>0
$$

and

$$
f(A x-A y) \leq \psi(f(x-y)) f(x-y) \text { for all } x, y \in K .
$$

In other words, the mapping $A$ is contractive [23]. We denote the identity operator by $A^{0}$.

In [22], we established the following result. 
Theorem 2.1. The mapping $A$ has a unique fixed point $x_{A} \in K$ and $A^{i} x \rightarrow x_{A}$ as $i \rightarrow \infty$ for all $x \in K$, uniformly on bounded subsets of $K$.

This result is also a generalization of the result of [24], which was obtained in the case where $f(x)=\|x\|$.

In this paper, we extend this result to a larger class of mappings. We also construct an example, which demonstrates that this new class is indeed larger than analogous classes of mappings which were studied in the literature.

\section{AN EXTENSION}

Assume that $(X,\|\cdot\|)$ is a Banach space, $K$ is a nonempty closed subset of $X$,

$$
A: K \rightarrow K
$$

and that $f: X \rightarrow[0, \infty)$ satisfies

$$
f(0)=0
$$

Let $x_{A} \in K$ satisfy

$$
A x_{A}=x_{A} .
$$

Assume that the following properties hold:

(P1)' for each $\varepsilon>0$, there exists $\delta>0$ such that if a point $x \in K$ satisfies $f\left(x-x_{A}\right) \leq \delta$, then $\left\|x-x_{A}\right\| \leq \varepsilon$

(P2)' the function $f\left(x-x_{A}\right), x \in D$, is bounded for every bounded set $D \subset K$.

Assume also that $\psi:[0, \infty) \rightarrow[0,1]$ is a decreasing function satisfying

$$
\psi(t)<1 \text { for all } t>0,
$$

and that

$$
f\left(A x-x_{A}\right) \leq \psi\left(f\left(x-x_{A}\right)\right) f\left(x-x_{A}\right) \text { for all } x \in K .
$$

We now prove the following result.

Theorem 3.1. $A^{n} x \rightarrow x_{A}$ as $n \rightarrow \infty$ for all $x \in K$, uniformly on bounded subsets of $K$.

Proof. Set $A^{0} x=x$ for all $x \in K$. Let $x \in K$. By (3.1), for every integer $n \geq 0$, we have

$$
\begin{aligned}
f\left(A^{n+1} x-x_{A}\right) & \leq \psi\left(f\left(A^{n} x-x_{A}\right)\right) f\left(A^{n} x-x_{A}\right) \\
& \leq f\left(A^{n} x-x_{A}\right) .
\end{aligned}
$$

Let $M, \varepsilon>0$ be given. Property (P1)' implies that there exists a number

$$
\delta \in(0, \varepsilon / 4)
$$

such that the following property holds:

(i) if $x \in K$ and $f\left(x-x_{A}\right) \leq 2 \delta$, then $\left\|x-x_{A}\right\| \leq \varepsilon / 2$.

Property (P2)' implies that there exists a number $M_{1}>0$ such that the following property holds:

(ii) if $x \in K$ satisfies $\left\|x-x_{A}\right\| \leq M$, then $f\left(x-x_{A}\right) \leq M_{1}$.

Choose an integer

$$
n_{0}>1+M_{1} \delta^{-1}(1-\psi(\delta))^{-1}
$$

and let a point $x \in K$ satisfy

$$
\left\|x-x_{A}\right\| \leq M
$$


We claim that there exists an integer $i \in\left[0, n_{0}\right]$ such that

$$
f\left(A^{i} x-x_{A}\right) \leq \delta
$$

Suppose to the contrary that this is not true. Then for each $i \in\left\{0, \ldots, n_{0}\right\}$, we have

$$
f\left(A^{i} x-x_{A}\right)>\delta
$$

Since the function $\psi$ is decreasing, it follows from (3.2) and (3.5) that, for each $i \in\left\{0, \ldots, n_{0}\right\}$,

$$
\begin{aligned}
f\left(A^{i+1} x-x_{A}\right) & \leq \psi\left(f\left(A^{i} x-x_{A}\right)\right) f\left(A^{i} x-x_{A}\right) \\
& \leq \psi(\delta) f\left(A^{i} x-x_{A}\right)
\end{aligned}
$$

and

$$
\begin{aligned}
f\left(A^{i} x-x_{A}\right)-f\left(A^{i+1} x-x_{A}\right) & \geq(1-\psi(\delta)) f\left(A^{i} x-x_{A}\right) \\
& \geq \delta(1-\psi(\delta)) .
\end{aligned}
$$

It now follows from (3.6) that

$$
\begin{aligned}
f\left(x-x_{A}\right) & \geq f\left(x-x_{A}\right)-f\left(A^{n_{0}} x-x_{A}\right) \\
& =\sum_{i=0}^{n_{0}-1}\left(f\left(A^{i} x-x_{A}\right)-f\left(A^{i+1} x-x_{A}\right)\right) \\
& \geq n_{0} \delta(1-\psi(\delta)) .
\end{aligned}
$$

Property (ii), (3.4) and (3.7) imply that

$$
\begin{aligned}
M_{1} & \geq f\left(x-x_{A}\right) \\
& \geq n_{0} \delta(1-\psi(\delta)),
\end{aligned}
$$

and

$$
n_{0} \leq M_{1} \delta^{-1}(1-\psi(\delta))^{-1}
$$

This, however, contradicts (3.3). The contradiction we have reached shows that there indeed exists an integer $j \in\left[0, n_{0}\right]$ such that

$$
f\left(A^{j} x-x_{A}\right) \leq \delta
$$

which together with (3.2) implies that

$$
f\left(A^{i} x-x_{A}\right) \leq \delta \text { for all integers } i \geq j .
$$

Property (i) and the above relation imply that, for all integers $i \geq n_{0}$,

$$
\left\|A^{i}-x_{A}\right\| \leq \varepsilon
$$

This completes the proof of this Theorem. 


\section{AN EXAMPLE}

Let $X=R^{2}$ be equipped with the Euclidean inner product which induces the Euclidean norm and let

$$
\begin{gathered}
K=\left\{x=\left(x_{1}, x_{2}\right) \in R^{2}: x_{i} \in[0,1], i=1,2\right\} \\
\cup\left\{\left(x_{1}, x_{2}\right) \in R^{2}: x_{1} \in[-1,0], x_{2} \in\left[-2^{-1}, 0\right]\right\} .
\end{gathered}
$$

Clearly, $K$ is a compact subset of $R^{2}$.

Let $x=\left(x_{1}, x_{2}\right) \in K$. Next, we define $A x \in R^{2}$. There are two cases:

$$
\begin{gathered}
x_{1}, x_{2} \in[0,1], \\
x_{1} \in[-1,0], x_{2} \in\left[-2^{-1}, 0\right] .
\end{gathered}
$$

First, assume that (4.1) holds and set

$$
A x:=\left(x_{2}, x_{1}\right) .
$$

If (4.2) is true, then we set

$$
A x:=\left(2 x_{2}, 2^{-1} x_{1}\right)
$$

By (4.1)-(4.4), $A x \in K$ in both cases. Clearly,

$$
A(0)=0 \text {. }
$$

Now, we define a function $f: R^{2} \rightarrow[0, \infty)$. If $x \in R^{2} \backslash K$, then we set $f(x):=0$.

Let $x=\left(x_{1}, x_{2}\right) \in K$. If $x_{1}, x_{2} \geq 0$, then we set

$$
f(x):=\max \left\{x_{1}, x_{2}\right\} .
$$

If $x_{1}, x_{2} \leq 0$, then we set

$$
f(x):=\max \left\{-2^{-1} x_{1},-x_{2}\right\} .
$$

It is easy to see that $f: R^{2} \rightarrow[0, \infty)$ is well defined and for all $x \in K$, we have

$$
f(A x-0)=f(A x)=f(x)=f(x-0) .
$$

Proposition 4.1. There is no norm $\|\cdot\|$ on $R^{2}$ such that for all $x \in K$,

$$
\|A x\| \leq\|x\| \text {. }
$$

Proof. Suppose to the contrary that $\|\cdot\|$ is a norm on $R^{2}$ such that (4.5) holds for all $x \in K$. By (4.1)-(4.4), we have

$$
\|(1,0)\| \geq\|A(1,0)\|=\|(0,1)\|,
$$

and

$$
\|(0,1)\| \geq\|A(0,1)\|=\|(1,0)\| .
$$

Thus

$$
\|(1,0)\|=\|(0,1)\| .
$$

In view of (4.1)-(4.4), we have

$$
\|(-1,0)\| \geq\|A(-1,0)\|=\left\|\left(0,-2^{-1}\right)\right\|,
$$

and

$$
\|\left(0,-2^{-1}\|\geq\| A\left(0,-2^{-1}\right)\|=\|(-1,0) \| .\right.
$$

Hence

$$
\|(-1,0)\|=\left\|\left(0,-2^{-1}\right)\right\| .
$$


This, however, contradicts (4.6). The contradiction we have reached completes the proof of this proposition.

Let $\gamma \in(0,1)$. Define a mapping $\gamma A: K \rightarrow K$ by

$$
(\gamma A)(x):=\gamma A x, x \in K
$$

It is easy to see that for all $x \in K$,

$$
(\gamma A)(x)=\gamma A x=A(\gamma x),
$$

and

$$
f((\gamma A) x)=f(A(\gamma x)) \leq f(\gamma x)=\gamma f(x) .
$$

Proposition 4.2. Let

$$
\gamma \in\left(2^{-1 / 2}, 1\right) .
$$

There is no norm $\|\cdot\|$ on $R^{2}$ such that

$$
\|\gamma A x\| \leq\|x\| \text { for all } x \in K .
$$

Proof. Suppose to the contrary that $\|\cdot\|$ is a norm on $R^{2}$ such that (4.8) holds. By (4.1)-(4.4) and (4.8), we have

$$
\begin{gathered}
\|(1,0)\| \geq\|\gamma A(1,0)\|=\gamma\|(0,1)\|, \\
\|(0,1)\| \geq\|\gamma A(0,1)\|=\gamma\|(1,0)\|, \\
\|(-1,0)\| \geq\|\gamma A(-1,0)\|=\gamma\left\|\left(0,-2^{-1}\right)\right\|,
\end{gathered}
$$

and

$$
\left\|\left(0,-2^{-1}\right)\right\| \geq\left\|\gamma A\left(0,-2^{-1}\right)\right\|=\gamma\|(-1,0)\| .
$$

These relations imply that

$$
\begin{gathered}
\gamma^{-1}\|(0,1)\| \geq\|(1,0)\| \geq \gamma\|(0,1)\|, \\
\gamma^{-1}\|(0,1)\| \geq\left\|\left(0,2^{-1}\right)\right\| \geq \gamma\|(1,0)\|,
\end{gathered}
$$

and

$$
2^{-1}\|(0,1)\|=\left\|\left(0,2^{-1}\right)\right\| \geq \gamma\|(1,0)\| \geq \gamma^{2}\|(0,1)\|
$$

Hence, we have

$$
2^{-1} \geq \gamma^{2}
$$

This, however, contradicts (4.7). The contradiction we have reached completes the proof of this proposition.

\section{Acknowledgments}

The first author was partially supported by the Israel Science Foundation (Grant No. 820/17), by the Fund for the Promotion of Research at the Technion and by the Technion General Research Fund. 


\section{REFERENCES}

[1] F. S. de Blasi, J. Myjak, S. Reich, A. J. Zaslavski, Generic existence and approximation of fixed points for nonexpansive set-valued maps, Set-Valued Var. Anal. 17 (2009), 97-112.

[2] K. Goebel, S. Reich, Uniform convexity, hyperbolic geometry, and nonexpansive mappings, Marcel Dekker, New York and Basel, 1984.

[3] A. Grzesik, W. Kaczor, T. Kuczumow, S. Reich, Convergence of iterates of nonexpansive mappings and orbits of nonexpansive semigroups, J. Math. Anal. Appl. 475 (2019), 519-531.

[4] E. Pustylnyk, S. Reich, A. J. Zaslavski, Convergence to compact sets of inexact orbits of nonexpansive mappings in Banach and metric spaces, Fixed Point Theory Appl. 2008 (2008), 1-10.

[5] S. Reich, A. J. Zaslavski, Generic aspects of metric fixed point theory, Handbook of Metric Fixed Point Theory, pp. 557-575, Kluwer, Dordrecht, 2001.

[6] S. Reich, A. J. Zaslavski, Convergence to attractors under perturbations, Commun. Math. Anal. 10 (2011), 57-63.

[7] S. Reich, A. J. Zaslavski, Convergence to attractors of nonexpansive set-valued mappings, Commun. Math. Anal. 22 (2019), 51-60.

[8] A. J. Zaslavski, Approximate solutions of common fixed point problems, Springer Optimization and Its Applications, Springer, Cham, 2016.

[9] A. J. Zaslavski, Algorithms for solving common fixed point problems, Springer Optimization and Its Applications, Springer, Cham, 2018.

[10] S. Banach, Sur les opérations dans les ensembles abstraits et leur application aux équations intégrales, Fund. Math. 3 (1922), 133-181.

[11] Y. Censor, M. Zaknoon, Algorithms and convergence results of projection methods for inconsistent feasibility problems: A review, Pure Appl. Func. Anal. 3 (2018), 565-586.

[12] A. Gibali, A new split inverse problem and an application to least intensity feasible solutions, Pure Appl. Funct. Anal. 2 (2017), 243-258.

[13] A. Gibali, S. Reich, R. Zalas, Outer approximation methods for solving variational inequalities in Hilbert space, Optimization 66 (2017), 417-437.

[14] O. Nevanlinna, S. Reich, Strong convergence of contraction semigroups and of iterative methods for accretive operators in Banach spaces, Israel J. Math. 32 (1979), 44-58.

[15] W. Takahashi, The split common fixed point problem and the shrinking projection method for new nonlinear mappings in two Banach spaces, Pure Appl. Funct. Anal. 2 (2017), 685-699.

[16] T.H. Cuong, J.C. Yao, N.D. Yen, Qualitative properties of the minimum sum-of-squares clustering problem, Optimization, 69 (2020), 2131-2154.

[17] S. Reich, A. J. Zaslavski, Contractivity and genericity results for a class of nonlinear mappings, J. Nonlinear Convex Anal. 16 (2015), 1113-1122.

[18] M. Gabour, S. Reich, A. J. Zaslavski, A generic fixed point theorem, Indian J. Math. 56 (2014), 25-32.

[19] F. S. de Blasi, J. Myjak, Sur la convergence des approximations successives pour les contractions non linéaires dans un espace de Banach, C. R. Acad. Sci. Paris 283 (1976), 185-187.

[20] S. Reich, A. J. Zaslavski, Well-posedness of generalized best approximation problems, Nonlinear Func. Anal. Appl. 7 (2002), 115-128.

[21] S. Reich, A. J. Zaslavski, Porous sets and generalized best approximation problems, Nonlinear Anal. Forum 9 (2004), 135-152.

[22] S. Reich, A. J. Zaslavski, Existence of a unique fixed point for nonlinear contractive mappings, Mathematics, 8 (2020), 55.

[23] S. Reich, A. J. Zaslavski, Genericity in nonlinear analysis, Developments in Mathematics, 34, Springer, New York, 2014.

[24] E. Rakotch, A note on contractive mappings, Proc. Amer. Math. Soc. 13 (1962), 459-465. 